Pawe€ BŁażej and JarosŁaw Bartoszewicz (Wrocław)

\title{
ROBUST ESTIMATION BASED ON SPACINGS IN WEIGHTED EXPONENTIAL MODELS
}

Abstract. Using Zieliński's $(1977,1983)$ formalization of robustness Błażej (2007) obtained uniformly most bias-robust estimates (UMBREs) of the scale parameter for some statistical models (including the exponential model), in a class of linear functions of order statistics, when violations of the models are generated by weight functions. In this paper the UMBRE of the scale parameter, based on spacings, in two weighted exponential models is derived. Extensions of results of Bartoszewicz $(1986,1987)$ are given.

1. Preliminaries. Let $X$ and $Y$ be two random variables, $F$ and $G$ their respective probability distribution functions, and $f$ and $g$ their respective density functions, if they exist. Denote by $\bar{F}=1-F$ the tail (or survival function) of $F$, by $F^{-1}(u)=\inf \{x: F(x) \geq u\}, u \in(0,1)$, the quantile (or reversed) function, and by $F^{-1}(0)$ and $F^{-1}(1)$ the lower and upper bounds of the support of $F$ respectively, and analogously for $G$. We identify the distribution functions $F$ and $G$ with the respective probability distributions and denote their supports by $S_{F}$ and $S_{G}$. We use increasing in place of nondecreasing and decreasing in place of nonincreasing.

1.1. Classes of life distributions and stochastic orders. A distribution $F$ is said to be IFR (DFR) [increasing (decreasing) failure rate] if $\log \bar{F}$ is concave (convex) on $S_{F}$ which is an interval.

2000 Mathematics Subject Classification: 60E15, 62F10, 62F35, 62 N02.

Key words and phrases: partial orders, weighted distributions, spacings, robustness, bias.

Research of P. Błażej supported by Ministry of Science and Higher Education, Poland, Grant N20104631/3733.

Research of J. Bartoszewicz supported by Ministry of Science and Higher Education, Poland, Grant 1 P03A 03629. 
An absolutely continuous distribution $F$ is said to be ILR (DLR) [increasing (decreasing) likelihood ratio] if $\log f$ is concave (convex) on $S_{F}$ which is an interval.

It is well known that

\section{$\mathrm{ILR} \subset \mathrm{IFR}$ and $\mathrm{DLR} \subset \mathrm{DFR}$.}

We deal with some stochastic orders. We recall their definitions and some properties for completeness. We use dual equivalent notations: for random variables and for distributions, as is usual in the literature.

We say that $X$ is smaller than $Y$ in the likelihood ratio order $\left(X \leq_{\operatorname{lr}} Y\right.$ or $\left.F \leq_{\operatorname{lr}} G\right)$ if $g(x) / f(x)$ is increasing.

We say that $X$ is smaller than $Y$ in the hazard rate order $\left(X \leq_{\mathrm{hr}} Y\right.$ or $\left.F \leq_{\text {hr }} G\right)$ if $\bar{G}(x) / \bar{F}(x)$ is increasing.

We say that $X$ is stochastically smaller than $Y\left(X \leq_{\mathrm{st}} Y\right.$ or $\left.F \leq_{\mathrm{st}} G\right)$ if $F(x) \geq G(x)$ for every $x$, or equivalently, if $\bar{F}(x) \leq \bar{G}(x)$ for every $x$. It is also well known that

$$
X \leq_{\operatorname{lr}} Y \Rightarrow X \leq_{\mathrm{hr}} Y \Rightarrow X \leq_{\mathrm{st}} Y .
$$

We say that $X$ is less dispersive than $Y\left(X \leq_{\operatorname{disp}} Y\right.$ or $\left.F \leq_{\operatorname{disp}} G\right)$ if

$$
F^{-1}(\beta)-F^{-1}(\alpha) \leq G^{-1}(\beta)-G^{-1}(\alpha) \text { whenever } 0<\alpha \leq \beta<1 \text {, }
$$

or equivalently, if $G^{-1} F(x)-x$ is increasing on $S_{F}$.

It is well known that if $-\infty<F^{-1}(0)=G^{-1}(0)$, then

$$
X \leq_{\text {disp }} Y \Rightarrow X \leq_{\text {st }} Y .
$$

Assume that a random variable $X$ has a continuous distribution function $F$. Denote by $X_{1: n}, X_{2: n}, \ldots, X_{n: n}$ the order statistics of a sample of size $n$ from distribution $F$. Put $X_{0: n}=F^{-1}(0)$ if it is finite. Then we define the random variables $V_{i: n}=X_{i: n}-X_{i-1: n}, i=1, \ldots, n$, called spacings from the distribution $F$. If $F^{-1}(0)$ is not finite, we define $V_{i: n}$ only for $i=2, \ldots, n$. Similarly we define the spacings $W_{i: n}$ of a sample of size $n$ from the distribution $G$.

We will use the following lemmas concerning relations between classes of distributions and stochastic orders.

Lemma 1 (Bartoszewicz, 1985). If $X \leq_{\mathrm{hr}} Y$ and $F$ or $G$ is DFR, then $X \leq_{\text {disp }} Y$.

Lemma $2(\mathrm{Oja}, 1981)$. Let $S_{F}=\left[0, a_{F}\right]$ and $S_{G}=\left[0, a_{G}\right]$, where $a_{F} \leq \infty$ and $a_{G} \leq \infty$. If $X \leq_{\text {disp }} Y$, then $V_{i: n} \leq_{\text {st }} W_{i: n}, i=1, \ldots, n$.

Lemma 3 (Barlow, Proschan, 1966). Let $F(0)=0$. If $F$ is IFR $(D F R)$, then $(n-i+1) E\left(V_{i: n}\right)$ is decreasing (increasing) in $i=1, \ldots, n$. 
For other properties of classes of life distributions and stochastic orders we refer to Barlow and Proschan (1975) and Shaked and Shanthikumar (2006).

1.2. Weighted distributions. Let $F$ be a distribution function, and let $w: \mathbb{R} \rightarrow \mathbb{R}^{+}$be such that $0<E[w(X)]<\infty$. Then

$$
F_{w}(x)=\frac{1}{E[w(X)]} \int_{0}^{x} w(z) d F(z)
$$

is the weighted distribution associated with $F$ with weight function $w$. The weighted distribution $F_{w}$ has density

$$
f_{w}(x)=\frac{w(x) f(x)}{E[w(X)]} .
$$

The idea of weighted distributions is due to Fisher (1934). Rao (1985) defined weighted distributions with a general weight function $w$. Patil and Rao $(1977,1978)$ provided some statistical models leading to weighted distributions and applied their results to the analysis of data relating to human population and ecology. Patil and Ord (1976) defined classes of distributions which are invariant under weighting with weight functions of type $x^{\alpha}, \alpha>0$.

Many authors, e.g. Jain et al. (1989), Bartoszewicz and Skolimowska (2006), studied preservation of classes of life distributions and stochastic orders under weighting. The following lemmas will be used. The first two of them are obvious.

Lemma 4. If the weight function $w$ is increasing, then $F \leq_{\mathrm{lr}} F_{w}$.

LEMMA 5. If $F$ is ILR (DLR) and $w$ is logconcave (logconvex), then $F_{w}$ is also ILR $(D L R)$.

Lemma 6 (Jain et al., 1989). If $F$ is IFR, $w$ is increasing and concave, then $F_{w}$ is IFR.

1.3. Robustness. Zieliński $(1977,1983)$ proposed the following formalization of robustness (see Box and Andersen, 1955). Let the original statistical model be $M_{0}=\left(\mathcal{X}, \mathcal{A}, \mathcal{P}_{0}\right)$, where $(\mathcal{X}, \mathcal{A})$ is a given measurable space and $\mathcal{P}_{0}$ is a given subset of the class $\mathcal{P}$ of all probability measures. Let $\pi: \mathcal{P}_{0} \rightarrow 2^{\mathcal{P}}$ be a function called a violation of $M_{0}$ which has the property that $P \in \pi(P)$, where $P \in \mathcal{P}_{0}$. Define $\mathcal{P}_{1}=\bigcup_{P \in \mathcal{P}_{0}} \pi(P)$. Thus $M_{1}=\left(\mathcal{X}, \mathcal{A}, \mathcal{P}_{1}\right)$ is an extension of the model $M_{0}$. Let $T$ be a suitable statistic with distribution $P^{T}(\cdot)=P\left(T^{-1}(\cdot)\right)$ and $\varrho$ be a real-valued function on $\mathcal{P}_{1}$. We have the following definitions.

Definition 1 (Zieliński, 1977). A function $r_{T}: \mathcal{P}_{0} \rightarrow \mathbb{R}^{+}$defined as

$$
r_{T}(P)=\sup \left\{\varrho\left(Q^{T}\right): Q \in \pi(P)\right\}-\inf \left\{\varrho\left(Q^{T}\right): Q \in \pi(P)\right\}
$$

is called the $\varrho$-robustness of the statistic $T$ in $M_{1}$. 
Definition 2 (Zieliński, 1977). A statistic $T^{0}$ is uniformly most @-robust in a given class $\mathcal{T}$ of statistics if

$$
r_{T^{0}}(P) \leq r_{T}(P) \quad \text { for every } P \in \mathcal{P}_{0} \text { and } T \in \mathcal{T} .
$$

In the recent paper Błażej (2007) derived the uniformly most bias-robust estimates (UMBREs) of the scale parameter for some statistical models (including the exponential model), in a class of nonnegative linear combinations of order statistics, when violations of the models are generated by weight functions. In this paper we consider the exponential model and two violations induced by monotone weight functions. Using properties of stochastic orders and weighted distributions we obtain the UMBREs of the scale parameter in the class of nonnegative linear combinations of spacings, larger than the previous one. The results are related to and extend those obtained by Bartoszewicz $(1986,1987)$ for a gamma violation of the exponential model.

2. Results. Let the original model be $M_{0}=\left(\mathbb{R}^{+}, \mathcal{B}^{+},\{F(\cdot ; \theta): \theta>0\}\right)$ where $F(\cdot ; \theta)$ is an exponential distribution with scale parameter $\theta$. Let $\mathcal{W}$

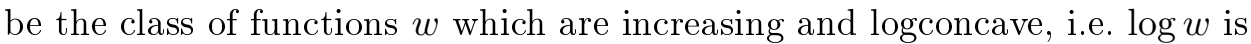
concave. Define

$$
\pi_{\mathcal{W}}(\theta)=\left\{F_{w}(\cdot ; \theta): w \in \mathcal{W}\right\}
$$

Notice that for every $\theta>0, \pi_{\mathcal{W}}(\theta)$ fulfils the following conditions:

(a) $F(\cdot ; \theta) \in \pi_{\mathcal{W}}(\theta)$;

(b) $\pi_{\mathcal{W}}\left(\theta^{\prime}\right) \cap\{F(\cdot ; \theta): \theta>0\}=\left\{F\left(\cdot ; \theta^{\prime}\right)\right\}$;

(c) $\pi_{\mathcal{W}}\left(\theta^{\prime}\right) \cap \pi_{\mathcal{W}}(\theta)=\emptyset$ for any $\theta^{\prime} \neq \theta$.

Condition (a) is obvious, conditions (b) and (c) follow from the fact that weight functions of the form $w(x) \exp (c x), c \neq 0, w \in \mathcal{W}$, are not in $\mathcal{W}$. Therefore

$$
M_{1}=\left(\mathbb{R}^{+}, \mathcal{B}^{+},\left\{\pi_{\mathcal{W}}(\theta): \theta>0\right\}\right)
$$

is an extension of $M_{0}$ under weighting. Our aim is to find a statistic $T^{0} \in \mathcal{T}^{+}$ which is the uniformly most bias-robust estimator (UMBRE) of the scale parameter $\theta$ with respect to the violation $\pi_{\mathcal{W}}(\theta), \theta>0$, of $M_{0}$, where

$$
\mathcal{T}^{+}=\left\{T=\sum_{j=1}^{n} a_{j} V_{j: n}: a_{j} \geq 0, j=1, \ldots, n ; E_{\theta}(T)=\theta, \theta>0\right\},
$$

i.e. $\mathcal{T}^{+}$is the class of those nonnegative linear combinations of spacings that are unbiased estimators of $\theta$ in $M_{0}$.

The following theorem holds.

THEOREM 1. Under the violation $\pi_{\mathcal{W}}(\theta), \theta>0$, given by (1) of the model $M_{0}$, the UMBRE of the scale parameter $\theta$ in the class $\mathcal{T}^{+}$is the statistic

$$
T^{0+}=V_{n: n} .
$$


Proof. Since $w \in \mathcal{W}$ is increasing, for every $\theta>0$ we have $F(\cdot ; \theta) \leq_{\operatorname{lr}}$ $F_{w}(\cdot ; \theta)$, which implies $F(\cdot ; \theta) \leq_{\mathrm{hr}} F_{w}(\cdot ; \theta)$. The exponential distribution is obviously DFR and so by Lemma $1, F(\cdot ; \theta) \leq_{\text {disp }} F_{w}(\cdot ; \theta)$. Hence from Lemma 2 and well known properties of the usual stochastic order it follows that for every $T \in \mathcal{T}^{+}$,

$$
\inf _{w \in \mathcal{W}} E_{w ; \theta}(T)=E_{\theta}(T), \quad \theta>0 .
$$

Since $E_{\theta}(T)=\theta E_{1}(T)$ for every $T \in \mathcal{T}^{+}$and every $\theta>0$, we obtain, for every $w \in \mathcal{W}$,

$$
\begin{aligned}
E_{w, \theta}(T)-E_{\theta}(T) & =\sum_{j=1}^{n} a_{j}\left[E_{w, \theta}\left(V_{j: n}\right)-E_{\theta}\left(V_{j: n}\right)\right] \\
& \geq \min _{1 \leq j \leq n} \frac{E_{w, \theta}\left(V_{j: n}\right)-E_{\theta}\left(V_{j: n}\right)}{1 /(n-j+1)}=\min _{1 \leq j \leq n} \frac{E_{w, \theta}\left(V_{j: n}\right)}{1 /(n-j+1)}-\theta .
\end{aligned}
$$

Since $F(\cdot ; \theta)$ is ILR, Lemma 5 shows that for every $w \in \mathcal{W}$ the distribution $F_{w}(\cdot ; \theta)$ is ILR and hence IFR. Then from Lemma 3 we obtain

$$
E_{w, \theta}(T)-E_{\theta}(T) \geq \frac{E_{w, \theta}\left(V_{n: n}\right)}{E_{1}\left(V_{n: n}\right)}-\theta=E_{w, \theta}\left(V_{n: n}\right)-\theta,
$$

and for every $T \in \mathcal{T}^{+}$and every $\theta>0$ we have

$$
\sup _{w \in \mathcal{W}}\left[E_{w, \theta}(T)-E_{\theta}(T)\right] \geq \sup _{w \in \mathcal{W}}\left[E_{w, \theta}\left(V_{n: n}\right)-\theta\right],
$$

which completes the proof.

Now let us consider the following class of weight functions. Let $w_{1}$ be a fixed positive decreasing and logconvex function (i.e. $\log w_{1}$ is convex), for example, $w_{1}(x)=x^{\alpha}, x>0,-1<\alpha<0$. Let

$$
\mathcal{W}_{1}=\left\{w: w \text { decreasing and } w / w_{1} \text { increasing }\right\} .
$$

Define the violation

$$
\pi_{\mathcal{W}_{1}}(\theta)=\left\{F_{w}(\cdot ; \theta): w \in \mathcal{W}_{1}\right\} .
$$

Notice that for every $\theta>0, \pi_{\mathcal{W}_{1}}(\theta)$ satisfies the following conditions:

(a) $F(\cdot ; \theta) \in \pi_{\mathcal{W}_{1}}(\theta)$;

(b) $\pi_{\mathcal{W}_{1}}\left(\theta^{\prime}\right) \cap\{F(\cdot ; \theta): \theta>0\}=\left\{F\left(\cdot ; \theta^{\prime}\right)\right\}$;

(c) $\pi_{\mathcal{W}_{1}}\left(\theta^{\prime}\right) \cap \pi_{\mathcal{W}_{1}}(\theta)=\emptyset$ for any $\theta^{\prime} \neq \theta$.

The following lemma holds.

Lemma 7. For every $w \in \mathcal{W}_{1}$ and every $\theta>0$,

$$
F_{w_{1}}(\cdot ; \theta) \leq_{\text {disp }} F_{w}(\cdot ; \theta) \leq_{\text {disp }} F(\cdot ; \theta) .
$$


Proof. Since $w / w_{1}$ is increasing, we have $F_{w_{1}}(\cdot ; \theta) \leq_{\operatorname{lr}} F_{w}(\cdot ; \theta)$ and so $F_{w_{1}}(\cdot ; \theta) \leq \mathrm{hr} F_{w}(\cdot ; \theta)$. By Lemma 5 the distribution $F_{w_{1}}(\cdot ; \theta)$ is DLR and hence DFR, and the first inequality in (3) follows from Lemma 1.

The other inequality may be proved similarly by noticing that $F_{w}(\cdot ; \theta) \leq_{\text {lr }}$ $F(\cdot ; \theta)$ and the exponential distribution is obviously DFR.

Now we can state the following result.

THEOREM 2. Under the violation $\pi_{\mathcal{W}_{1}}(\theta), \theta>0$, given by (2) of the model $M_{0}$, the UMBRE of the scale parameter $\theta$ in the class $\mathcal{T}^{+}$is the statistic

$$
T^{0+}=V_{n: n} .
$$

Proof. The proof is similar to that of Theorem 1. From Lemmas 7 and 2 and properties of the usual stochastic order it follows that

$$
\sup _{w \in \mathcal{W}_{1}} E_{w, \theta}(T)=E_{\theta}(T)=\theta, \quad \theta>0 .
$$

Since $F_{w_{1}}(\cdot ; \theta)$ is DFR, directly from Lemma 3 we obtain, for every $\theta>0$,

$$
\sup _{w \in \mathcal{W}_{1}}\left[\theta-E_{w_{1}, \theta}(T)\right] \geq \sup _{w \in \mathcal{W}_{1}}\left[E_{\theta}\left(V_{n: n}\right)-E_{w_{1}, \theta}\left(V_{n: n}\right)\right]
$$

which completes the proof.

\section{References}

R. E. Barlow and F. Proschan (1966), Inequalities for linear combinations of order statistics from restricted families, Ann. Math. Statist. 37, 1574-1592.

R. E. Barlow and F. Proschan (1975), Statistical Theory of Reliability and Life Testing, Holt, Rinehart and Winston, New York.

J. Bartoszewicz (1985), Dispersive ordering and monotone failure rate distributions, Adv. Appl. Probab. 17, 472-474.

J. Bartoszewicz (1986), Bias-robust estimation of scale parameter, Probab. Math. Statist. 7, 103-113.

J. Bartoszewicz (1987), Bias-robust estimates based on order statistics and spacings in the exponential model, Zastos. Mat. 19, 57-63.

J. Bartoszewicz and M. Skolimowska (2006), Preservation of classes of life distributions and stochastic orders under weighting, Statist. Probab. Lett. 76, 587-596.

P. Błażej (2007), Robust estimation of the scale and weighted distributions. Appl. Math. (Warsaw) 34, 39-45.

G. P. Box and S. L. Andersen (1955), Permutation theory in the derivation of robust criteria and the study of departures from assumption, J. Roy. Statist. Soc. Ser. B 17, $1-34$.

R. A. Fisher (1934), The effects of methods of ascertainment upon the estimation of frequencies, Ann. Eugenics 6, 13-25.

K. Jain, H. Singh and I. Bagai (1989), Relations for reliability measures of weighted distributions, Comm. Statist. Theory Methods 18, 4393-4412.

H. Oja (1981), On location, scale, skewness and kurtosis of univariate distributions, Scand. J. Statist. 8, 154-168. 
G. P. Patil and J. K. Ord (1976), On size-biased sampling and related form invariant weighted distributions, Sankhyā Ser. B 38, 48-61.

G. P. Patil and C. R. Rao (1977), The weighted distributions: A survey and their applications, in: Applications of Statistics, P. R. Krishnaiah (ed.), North-Holland, Amsterdam, 383-405.

G. P. Patil and C. R. Rao (1978), Weighted distributions and size biased sampling with applications to wild-life populations and human families, Biometrica 34, 179-189.

C. R. Rao (1985), Weighted distributions arising out of methods of ascertainment: What population does a sample represent?, in: Celebration of Statistics: The ISI Centenary Volume, A. C. Atkinson and S. E. Fienberg (eds.), Springer, New York, 543-569.

M. Shaked and J. G. Shanthikumar (2006), Stochastic Orders, Springer, New York.

R. Zieliński (1977), Robustness: a quantitative approach, Bull. Acad. Polon. Sci. Sér. Sci. Math. Astronom. Phys. 25, 1281-1286.

R. Zieliński (1983), Robust statistical procedures: A general approach, in: Lecture Notes in Math. 982, Springer, Berlin, 283-295.

Mathematical Institute

University of Wrocław

Pl. Grunwaldzki $2 / 4$

50-384 Wrocław, Poland

E-mail: blazej@math.uni.wroc.pl jarbar@math.uni.wroc.pl

Received on 22.11.2007;

revised version on 13.12.2007 
